\title{
Global stability of enzymatic chains of full reversible Michaelis-Menten reactions
}

\author{
Ismail Belgacem · Jean-Luc Gouzé
}

Received: date / Accepted: date

\begin{abstract}
We consider a chain of metabolic reactions catalyzed by enzymes, of reversible Michaelis-Menten type with full dynamics, i.e. not reduced with any quasisteady state approximations. We study the corresponding dynamical system and show its global stability if the equilibrium exists. If the system is open, the equilibrium may not exist. The main tool is monotone systems theory. Finally we study the implications of these results for the study of coupled genetic-metabolic systems.
\end{abstract}

Keywords Biological systems · Dynamical Systems · Nonlinear systems · Global Stability $\cdot$ Enzymatic systems $\cdot$ Michaelis-Menten equation

\section{Introduction}

In this paper, we study the dynamical behaviour of a metabolic chain with enzyme regulation, made of $n$ enzymatic reactions; this configuration is classical in biological networks (Stephanopoulos et al, 1998). The most famous and classical enzymatic system is the (irreversible or reversible) Michaelis-Menten system. In particular, the study of the reduced system of Michaelis-Menten with the QSSA (Quasi-Steady State Approximation), based on the difference between the time scales of the reactions, led to thousand of studies (Segel and Slemrod, 1989; Heinrich and Schuster, 1996). These studies mainly compare the behavior of the full system and that of the reduced one.

Yet, the study of the full Michaelis-Menten system stability is of great interest because this system is often used as a fast metabolic system coupled with a slow

This work was supported by ANR Gemco project, and INRIA/INSERM Colage action. A part of these results was presented during IFAC ADCHEM 2012 conference.

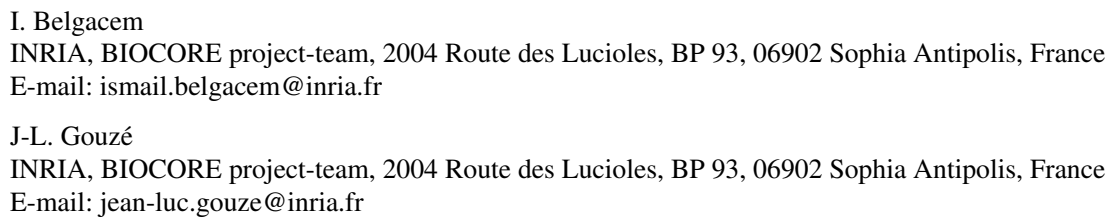


genetic system (Steuer and Junker, 2008) coding for the enzymes. The global stability of the metabolic part allows to apply QSSA methods to reduce the whole system. This study also gives conditions when the QSSA methods are not applicable, if, for example, the equilibrium of the metabolic system does not exist.

Many papers have studied Michaelis-Menten approximations, but few are interested in the full system, in a chain or not. The paper (Fraser and Roussel, 1994) studies the full closed reversible Michaelis-Menten by reducing it to a two-dimensional system. There exists other studies of metabolic chains controlled by enzymes, in other contexts (Oyarzún et al, 2012), but, to our knowledge, none of them with our tools. For an application of monotone systems theory for chemical chains, see (De Leenheer et al, 2007). For a work on a similar problem of metabolic chains, with a linear approach, see (Flach and Schnell, 2010). Other works on metabolic chains exist, but use the reduced classical Michaelis-Menten approximation (Chitour et al, 2006) or compare several approximations (Ciliberto et al, 2007). We believe that our tools are well adapted to biological problems, as noticed by other studies (Sontag, 2004).

In this paper, we study the dynamical behavior of the full metabolic chain without any approximation; we use some of the results of (Belgacem and Gouzé, 2012). We show, with simple mathematical tools, the stability of the unique equilibrium of the closed or open reversible chain. But we also show that the open reversible MichaelisMenten chain may have no equilibrium, if the input is too large. The mathematical tools we use are known but not so classical: they belong to the theory of monotone systems, and of compartmental systems. From this point of view, we continue the work done in (Ndiaye and Gouzé, 2013) for metabolic chains or networks with reduced enzymatic kinetics.

In the last section, we briefly give some conclusions about the coupled metabolicgenetic system. The mathematical notions and theorems used in this paper are briefly recalled in the appendix.

Notations: First we give some classical notations (see (Perko, 2001)). Consider the autonomous n-dimensional differential system

$$
\dot{x}=f(x)
$$

The notation $x\left(t, x_{1}\right)$, or briefly $x(t)$, corresponds to the solution starting from the initial condition $x_{1}$ parametrized by time $t \geq 0$. Function $f$ is supposed to be continuously differentiable within some domain of interest, that will be in our case $X=\mathfrak{R}_{+}^{n}$. We deduce the existence and uniqueness of solutions on some time interval for the differential equation (1). Throughout the paper, we use the classical notions of Lyapunov stability.

\section{The closed full reversible Michaelis-Menten system}

For completeness, we summarize in this section some results of (Belgacem and Gouzé, 2012). In principle,(see (Cornish-Bowden, 2004)), all reactions catalyzed by enzymes are reversible (similarly to chemical reactions), and this fact could play a prominent role in biochemistry. Michaelis and Menten proposed the following description and equations (we refer to (Murray, 2002) and (Edelstein-Keshet, 1988)). The enzyme 
forms a transitory complex $C$ before returning to its original form, giving product $P$ from substrate $S$. The last step being reversible, the classical reaction scheme is:

$$
S+E \underset{k_{-1}}{\stackrel{k_{1}}{\rightleftharpoons}} C \underset{k_{-2}}{\stackrel{k_{2}}{\rightleftharpoons}} P+E
$$

The mathematical model, based on classical mass-actions chemical laws, writes:

$$
\left\{\begin{array}{l}
\frac{d s}{d t}=-k_{1} s e+k_{-1} c \\
\frac{d e}{d t}=-k_{1} s e+k_{-1} c+k_{2} c-k_{-2} p e \\
\frac{d c}{d t}=k_{1} s e-k_{-1} c-k_{2} c+k_{-2} p e \\
\frac{d p}{d t}=k_{2} c-k_{-2} p e
\end{array}\right.
$$

with the two conservation laws:

$$
\dot{e}+\dot{c}=0, \dot{s}+\dot{c}+\dot{p}=0
$$

This is the model of the full reversible Michaelis-Menten system, that we want to study without any approximation or reduction. In a first step, we remark that the vector field is repulsive on the boundary of the positive orthant; thus for nonnegative initial conditions, the variables stay positive for all time $t$.

The sum of the free form and bound form of the enzyme is constant, thus we can write $e+c=E_{0}$, where $E_{0}$ is a positive constant, which is the total concentration of enzyme. Therefore we can eliminate the state variable $e=E_{0}-c$, and obtain the constraint that $c(t) \leq E_{0}$ has to be fulfilled for all time $t$.

The reduced system is :

$$
\left\{\begin{array}{l}
\frac{d s}{d t}=-k_{1} s\left(E_{0}-c\right)+k_{-1} c \\
\frac{d c}{d t}=k_{1} s\left(E_{0}-c\right)-k_{-1} c-k_{2} c+k_{-2} p\left(E_{0}-c\right) \\
\frac{d p}{d t}=k_{2} c-k_{-2} p\left(E_{0}-c\right)
\end{array}\right.
$$

Positive equilibrium. The first problem in this case is to show that the equilibrium always exists and fulfills the constraints on the variables that $\left(c^{*}<E_{0}\right)$ :

System (3) is closed. Thus if we take $M_{0}>0$ such that $M(s, c, p)=s+c+p=M_{0}$ then we obtain at equilibrium :

$$
\begin{aligned}
& s^{*}+c^{*}+p^{*}=M_{0} \\
& \Rightarrow \frac{k_{-1} c^{*}}{k_{1}\left(E_{0}-c^{*}\right)}+c^{*}+\frac{k_{2} c^{*}}{k_{-2}\left(E_{0}-c^{*}\right)}=M_{0}
\end{aligned}
$$

Note that the left side of (4) is an increasing function of $c^{*}$, which is zero for $c^{*}$ equals to zero, and tends to infinity when $c^{*}$ tends toward $E_{0}$. Thus, for a fixed $M_{0}$, we deduce that (4) has a unique solution $c^{*}$ such that $0<c^{*}<E_{0}$ and which depends 
on hyperplane $H_{0}=\left\{(s, c, p) \in \mathfrak{R}_{+}^{3}: s+c+p+=M_{0}>0\right\}$. The whole steady state can be obtained by solving (4) for $c^{*}$.

The Jacobian of the system (3) matrix is $J(s, c, p)$

$$
=\left(\begin{array}{ccc}
-k_{1}\left(E_{0}-c\right) & k_{-1}+k_{1} s & 0 \\
k_{1}\left(E_{0}-c\right) & -k_{-1}-k_{1} s-k_{2}-k_{-2} p & k_{-2}\left(E_{0}-c\right) \\
0 & k_{2}+k_{-2} p & -k_{-2}\left(E_{0}-c\right)
\end{array}\right)
$$

This matrix is compartmental. We recall in the appendix the definition of a compartmental matrix. This matrix is also irreducible and we obtain (see (Belgacem and Gouzé, 2012))

Proposition 1 In the invariant hyperplane $M_{0}=s+c+p$, the equilibrium of (3) is unique, positive, and globally asymptotically stable.

Remark 1. If the last step of the reaction is irreversible $\left(k_{-2}=0\right)$, then the equilibrium is $s^{*}=c^{*}=0, p^{*}=M_{0}$. The Jacobian matrix is not irreducible, but the global stability can still be proven.

For $c=E_{0}$ (and a reversible reaction), the Jacobian matrix is not irreducible neither. But in this case $\dot{c}=-k_{-1} E_{0}-k_{2} E_{0}$ is strictly negative (this point corresponds to $e=0$ ), therefore the point is never attained.

Remark 2. The study of a closed chain of $n$ such reactions is possible with the same tools and the same results.

\section{Open enzymatic chain with one input and output}

In Belgacem and Gouzé (2012), we have proven the global stability for a single full reversible Michaelis-Menten reaction; here we consider a chain of such reactions. For the first reaction, there is an input $u$ for substrate, coming from "outside", and for the last reaction an output for the final product, taken as a linear decay rate. The existence of such metabolic chains in biological networks are classical (Stephanopoulos et al, 1998). In the following we aim to apply our tools to this chain. To simplify the computations we take $n=3$ but the results are the same for general $n$. The reaction scheme of the chain is as follows :

$$
u \longrightarrow S_{1}+E_{1} \underset{k_{-11}}{\stackrel{k_{11}}{\rightleftharpoons}} C_{1} \underset{\substack{k_{-12} \\ \underset{E_{1}}{\rightleftharpoons}}}{\stackrel{k_{12}}{\rightleftharpoons}} S_{2}+E_{2} \underset{k_{-21}}{\stackrel{k_{21}}{\rightleftharpoons}} C_{2} \underset{k_{-22}}{\stackrel{k_{22}}{\rightleftharpoons}} E_{2}+S_{3} \longrightarrow
$$

As before, we use the conservation laws to set $e_{1}=\left(E_{01}-c_{1}\right)$ and $e_{2}=\left(E_{02}-c_{2}\right)$ and obtain:

$$
\left\{\begin{array}{l}
\dot{s_{1}}=u-k_{11} s_{1}\left(E_{01}-c_{1}\right)+k_{-11} c_{1} \\
\dot{c_{1}}=k_{11} s_{1}\left(E_{01}-c_{1}\right)-k_{-11} c_{1}-k_{12} c_{1}+k_{-12} s_{2}\left(E_{01}-c_{1}\right) \\
\dot{s_{2}}=k_{12} c_{1}-k_{-12} s_{2}\left(E_{01}-c_{1}\right)-k_{21} s_{2}\left(E_{02}-c_{2}\right)+k_{-21} c_{2} \\
\dot{c_{2}}=k_{21} s_{2}\left(E_{02}-c_{2}\right)-k_{-21} c_{2}-k_{22} c_{2}+k_{-22} s_{3}\left(E_{02}-c_{2}\right) \\
\dot{s_{3}}=k_{22} c_{2}-k_{-22} s_{3}\left(E_{02}-c_{2}\right)-k s_{3}
\end{array}\right.
$$


The last term $-k s_{3}$ represents the output of the last substrate.

Proposition 2 There exists a positive unique equilibrium $\left(s_{1}^{*}, c_{1}^{*}, s_{2}^{*}, c_{2}^{*}, s_{3}^{*}\right)$ if and only if $u<k_{i 2} E_{0 i}$ for $i=1,2$.

Proof Note that at the equilibrium we have for $i=1,2$ :

$$
\left\{\begin{array}{l}
u-k_{i 1} s_{i}^{*}\left(E_{0 i}-c_{i}^{*}\right)+k_{-i 1} c_{i}^{*}=0 \\
u-k_{i 2} c_{i}^{*}+k_{-i 2} s_{i+1}^{*}\left(E_{0 i}-c_{i}^{*}\right)=0 \quad c_{i}^{*}<E_{0 i}
\end{array}\right.
$$

and

$$
u=k s_{3}^{*}
$$

Therefore for $i=1,2$ :

$$
\left\{\begin{array}{l}
s_{i}^{*}=\frac{u+k_{-i 1} c_{i}^{*}}{k_{i 1}\left(E_{0 i}-c_{i}^{*}\right)} \\
c_{i}^{*}=\frac{u+k_{-i 2} s_{i+1}^{*} E_{0 i}}{k_{-i 2} s_{i+1}^{*}+k_{i 2}}
\end{array}\right.
$$

and

$$
\begin{gathered}
s_{3}^{*}=\frac{u}{k} \\
c_{2}^{*}=\frac{u+k_{-22} s_{3}^{*} E_{02}}{k_{-22} s_{3}^{*}+k_{22}}=\frac{\left(1+\frac{k_{-22} E_{02}}{k}\right) u}{\frac{k_{-22}}{k} u+k_{22}}
\end{gathered}
$$

This function of $u$ is strictly increasing from 0 (for $u=0$ ) to $E_{02}+\frac{k}{k_{-22}}$ when $u$ tends to infinity. The constraint $c_{2}^{*}<E_{02}$ gives $\frac{\left(1+\frac{k_{-22} E_{02}}{k}\right) u}{\frac{k_{-22}}{k} u+k_{22}}<E_{02}$ which implies $u<$ $k_{22} E_{02}$. If this inequality is fulfilled, we can compute the value of $s_{2}^{*}=\frac{u+k_{-21} c_{2}^{*}(u)}{k_{21}\left(E_{02}-c_{2}^{*}(u)\right)}$.

Similarly we can also compute $c_{1}^{*}$ from (9) with the value of $s_{2}^{*}(u)$ from above. Remember that $c_{2}^{*}(u)=0$ if $u=0$, therefore $c_{1}^{*}(0)=0$. Moreover the function $c_{1}^{*}(u)$ is increasing as a composite function of increasing functions. We also have the constraint $c_{1}^{*}<E_{01}$, so as previously we conclude from expression of $c_{1}^{*}$ in (9) that $u<k_{12} E_{01}$, which ends the proof.

In the following the aim is to study the global stability of the equilibrium when $u<k_{12} E_{01}$, and $u<k_{22} E_{02}$, i.e. the case where the equilibrium exists.

Proposition 3 If for $i=1,2: u<k_{i 2} E_{0 i}$, then the equilibrium exists and is globally stable in the invariant hyperrectangle defined by $(0,0,0,0,0)$ as the lower corner, and $\left(s_{1}, c_{1}, s_{2}, c_{2}, s_{3}\right)$ as the upper corner, such that : $c_{1}=E_{01}-e_{1}, c_{2}=E_{02}-e_{2}$ with $e_{1}, e_{2}$ small enough to satisfy:

1. $k_{12}\left(E_{01}-e_{1}\right)-u>0$

2. $\left.k_{22}\left(E_{02}-e_{2}\right)-u\right)>0$ 
The coordinates of this upper corner point are:

$$
\begin{gathered}
e_{2}=\frac{\left(u+k_{-21} E_{02}\right) k_{-12} e_{1}}{k_{21}\left(k_{12}\left(E_{01}-e_{1}\right)-u\right)+k_{-21} k_{-12} e_{1}} \\
s_{1}=\frac{u+k_{-11}\left(E_{01}-e_{1}\right)}{k_{11} e_{1}} ; s_{2}=\frac{k_{12}\left(E_{01}-e_{1}\right)-u}{k_{-12} e_{1}}=\frac{u+k_{-21}\left(E_{02}-e_{2}\right)}{k_{21} e_{2}} ; s_{3}=\frac{k_{22}\left(E_{02}-e_{2}\right)-u}{k_{-22} s_{3} e_{2}} .
\end{gathered}
$$

Note that $s_{1}, s_{2}, s_{3}$ tend to infinity when $e_{1}$ tends to 0 and $e_{2}$ tends to 0 . Moreover, the equilibrium is locally exponentially asymptotically stable.

Proof The Jacobian matrix of system (6) is still compartmental, as it is easy to check; so we can also investigate the stability of the equilibrium when $u<k_{12} E_{01}$ and $u<k_{22} E_{02}$ (which are the conditions for existence of equilibrium) by using the property (4) of monotone systems (cf. appendix). For a monotone system, $f$ there is a point greater than the equilibrium point (with respect to the usual partial ordering) where all the derivatives are nonpositive and similarly, if there is a point smaller than the equilibrium where the derivatives are nonnegative, then the whole hyperrectangle built with these two points is invariant, and all the trajectories initiating in this rectangle converge toward the equilibrium.

Thus we have to find the two extreme vertices of the rectangle. For the lower one, it is easy ; we choose $s_{1}=c_{1}=s_{2}=c_{2}=s_{3}=0$ and obtain that the following vector field

$$
\dot{s}_{1}=u ; \dot{c}_{1}=0 ; \dot{s}_{2}=0 ; \dot{c}_{2}=0 ; \dot{s}_{3}=0
$$

which is nonnegative.

For the upper vertex, we write the inequality we wish to fulfill:

$$
\begin{aligned}
& u-k_{11} s_{1}\left(E_{01}-c_{1}\right)+k_{-11} c_{1} \leq 0 \\
& k_{11} s_{1}\left(E_{01}-c_{1}\right)-k_{-11} c_{1}-k_{12} c_{1}+k_{-12} s_{2}\left(E_{01}-c_{1}\right) \leq 0 \\
& k_{12} c_{1}-k_{-12} s_{2}\left(E_{01}-c_{1}\right)-k_{21} s_{2}\left(E_{02}-c_{2}\right)+k_{-21} c_{2} \leq 0 \\
& k_{21} s_{2}\left(E_{02}-c_{2}\right)-k_{-21} c_{2}-k_{22} c_{2}+k_{-22} s_{3}\left(E_{02}-c_{2}\right) \leq 0 \\
& k_{22} c_{2}-k_{-22} s_{3}\left(E_{02}-c_{2}\right)-k_{3} \leq 0
\end{aligned}
$$

Firstly, to obtain a basin of attraction as large as possible we fixed $c_{1}$ a little bit smaller than $E_{01}\left(c_{1}=E_{01}-e_{1}\right)$ and $c_{2}$ a little bit smaller than $E_{02}\left(c_{2}=E_{02}-e_{2}\right)$. We firstly study necessary conditions. Summing the first four inequalities and taking $c_{2}=E_{02}-e_{2}$, we obtain :

$$
u-k_{22}\left(E_{02}-e_{2}\right)+k_{-22} s_{3}\left(E_{02}-c_{2}\right) \leq 0 \Rightarrow s_{3} \leq \frac{k_{22}\left(E_{02}-e_{2}\right)-u}{k_{-22} e_{2}} .
$$

We know that $k_{22} E_{02}-u>0$ because the equilibrium exists, therefore we can choose $s_{3}=\frac{k_{22}\left(E_{02}-e_{2}\right)-u}{k_{-22} s_{3} e_{2}}$ with $e_{2}$ small enough to fulfill $k_{22} E_{02}-u-k_{22} e_{2}>0$.

Similarly summing the first three inequalities and taking $c_{2}=E_{02}-e_{2}$ we have :

$$
u-k_{21} s_{2} e_{2}+k_{-21}\left(E_{02}-e_{2}\right) \leq 0 \Rightarrow s_{2} \geq \frac{u+k_{-21}\left(E_{02}-e_{2}\right)}{k_{21} e_{2}}
$$




$$
\text { we choose } s_{2}=\frac{u+k_{-21}\left(E_{02}-e_{2}\right)}{k_{21} e_{2}} .
$$

Summing also the first two inequalities and taking $c_{1}=E_{01}-e_{1}$ we have :

$$
\begin{gathered}
u-k_{12}\left(E_{01}-e_{1}\right)+k_{-12} s_{2} e_{1} \leq 0 \Rightarrow s_{2} \leq \frac{k_{12}\left(E_{01}-e_{1}\right)-u}{k_{-12} e_{1}} \\
\text { then we choose } s_{2}=\frac{k_{12}\left(E_{01}-e_{1}\right)-u}{k_{-12} e_{1}}
\end{gathered}
$$

We also know that $k_{2} E_{0}-u>0$, thus we take $e_{1}$ small enough to have $k_{12}\left(E_{01}-\right.$ $\left.e_{1}\right)-u>0$. From the equations (13) and (15) we can write :

$$
s_{2}=\frac{k_{12}\left(E_{01}-e_{1}\right)-u}{k_{-12} e_{1}}=\frac{u+k_{-21}\left(E_{02}-e_{2}\right)}{k_{21} e_{2}} .
$$

therefore we can compute $e_{2}$ as a function of $e_{1}$

$$
e_{2}=\frac{\left(u+k_{-21} E_{02}\right) k_{-12} e_{1}}{k_{21}\left(k_{12}\left(E_{01}-e_{1}\right)-u\right)+k_{-21} k_{-12} e_{1}}
$$

It is easy to check with this equation that $e_{2}$ tends to 0 when $e_{1}$ tends to zero. Therefore it is possible to choose $e_{1}$ and $e_{2}$ both small enough to check the constraints 1 . and 2. of the theorem. Then we can choose:

$$
s_{1}=\frac{u+k_{-11}\left(E_{01}-e_{1}\right)}{k_{11} e_{1}} ; s_{2}=\frac{k_{12}\left(E_{01}-e_{1}\right)-u}{k_{-12} e_{1}}=\frac{u+k_{-21}\left(E_{02}-e_{2}\right)}{k_{21} e_{2}} ; s_{3}=\frac{k_{22}\left(E_{02}-e_{2}\right)-u}{k_{-22} s_{3} e_{2}}
$$

Note that when $e_{1}$ tends to 0 and $e_{2}$ tends to $0, s_{1}, s_{2}$, and $s_{3}$ tends to infinity; we obtain the following vector field $\dot{s_{1}}=0, \dot{c_{1}}=0, \dot{s_{2}}=0, \dot{c_{2}}=0$ and $\dot{s_{3}} \rightarrow-\infty$. This vector field is nonpositive, as desired.

In conclusion we obtain the global stability in the hyperrectangle with the upper and lower vertices given above. Concerning the exponential stability, it is easy to check that the Jacobian matrix around the equilibrium has a graph which is outflowconnected (see appendix), and therefore the matrix is bijective and asymptotically stable.

Remark 3. If the conditions of Proposition ?? are not fulfilled, an equilibrium does not exist; it is the case, for example, when input $u$ is too large with respect to $E_{0 i}$. Then it is easy to check that at least one of the two substrates $s_{i}$ will tend to infinity.

Simulations. We next performed simulations for the case when the equilibrium exists $\left(u<k_{12} E_{01}, u<k_{22} E_{02}\right)$ and a case when the equilibrium does not exist $\left(k_{12} E_{01}<\right.$ $u<k_{22} E_{02}$ ). The results of simulations are shown in the figures (1) and (2). As predicted, in the first case, the system is stable and converges to the equilibrium, whereas, in the second case, it is not stable and the concentration of first substrate $S_{1}$ grows to infinity. 
Fig. 1 The simulation of system (6) for $u<k_{12} E_{01}$ and $u<k_{22} E_{02}$, with the following parameters values $k_{11}=0.2, k_{-11}=0.2, k_{12}=0.4, k_{-12}=0.2, k_{21}=0.3, k-21=0.2, k_{22}=0.4, k_{-22}=0.2, k=0.1, E_{01}=$ $1, E_{02}=1, u=0.2, S_{01}=70, S_{02}=0.8, S_{03}=20, S_{04}=0.7, S_{05}=15$. The trajectory converges toward the equilibrium.

Fig. 2 The simulation of system (6) for $k_{12} E_{01}<u<k_{22} E_{02}$, with the following parameters values $k_{11}=$ $0.2, k_{-11}=0.2, k_{12}=0.2, k_{-12}=0.2, k_{21}=0.3, k-21=0.2, k_{22}=0.4, k_{-22}=0.2, k=0.1, E_{01}=1, E_{02}=$ $1, u=0.3, S_{01}=35, S_{02}=0.8, S_{03}=28, S_{04}=0.7, S_{05}=20$. There is no equilibrium and substrate $s_{1}$ grows to infinity.

\section{Coupling metabolic and genetic systems}

The metabolic enzymatic chain reactions are biochemical reactions whose final product can influence the production of enzymes via gene regulation. These cross interactions between genetic and metabolic systems form a coupled system controlled by feedback loops (Goelzer et al, 2008). Let's consider a coupled metabolic genetic system defined by the following equations :

$$
\dot{s}=f(s, e) ; \dot{e}=g(s, e)
$$

where $s$ is the vector of metabolite concentrations, $e$ is the vector of enzyme concentrations (see Fig.3).

For example, if an end-product $s_{n}$ of the metabolic chain represses the expression of the gene associated to the enzyme $e_{1}$, the equation could classically be:

$$
\dot{e}_{1}=\alpha h^{-}\left(s_{n}\right)-\gamma e_{1}
$$

where the first term represents the repression $\left(h^{-}\right.$is a decreasing function) and the second term is a linear unregulated degradation.

Fig. 3 A metabolic chain with enzymatic regulation; the product of the chain represses the expression of the enzyme catalyzing the first step of the chain

The dynamic of the metabolic system is very fast compared to the genetic system. If the metabolic system is globally stable, it will go very quickly to the equilibrium point $s^{*}$ where we can apply the QSSA hypotheses leading to $s^{*}=\varphi(e)$. The genetic system then becomes :

$$
\dot{e}=g(\varphi(e), e)
$$

Importantly this time-scale reduction is valid only if the metabolic system is globally stable, therefore it is necessary to check if the equilibrium of the metabolic system exists. If $u<k_{i 2} E_{0 i}$ for all $i=1, . ., n-1$, according to our previous results, the good hypotheses are fulfilled, the metabolic system is stable, and the QSSA reduction can apply. But if one of the above inequality is not fulfilled, the equilibrium does not exist, and the QSSA reduction does not apply. Because the enzyme concentration $e_{i}$ 
are now dynamical variables, it may happen that, at the beginning, the equilibrium exists, but disappears after some time because one of the $e_{i}$ became too small.

\section{Conclusion}

We were able to show stability of a full reversible Michaelis Menten chain. For the open systems, we showed that if the input $u$ is too large, there is no equilibrium. We think that these kind of tools could be applied to other metabolic systems, and to coupled metabolic/genetic systems. The generalization of these results concerning stability to enzymatic networks (and not only chains) should be possible.

\section{References}

Bastin G, Guffens V (2006) Congestion control in compartmental network systems. Systems and Control Letters 55:689-696

Belgacem I, Gouzé JL (2012) Global stability of full open reversible michaelismenten reactions. In: 8th IFAC Symposium Advanced Control of Chemical Processes ADCHEM, Singapore, pp 591-596

Chitour Y, Grognard F, Bastin G (2006) Equilibria and stability analysis of a branched metabolic network with feedback inhibition. Networks and Heterogeneous Media 1(1):219

Ciliberto A, Capuani F, Tyson J (2007) Modeling networks of coupled enzymatic reactions using the total quasi-steady state approximation. PLoS computational biology 3(3):e45

Cornish-Bowden A (2004) Fundamentals of enzyme kinetics, vol 3. Portland Press London

De Leenheer P, Angeli D, Sontag E (2007) Monotone chemical reaction networks. Journal of mathematical chemistry 41(3):295-314

Edelstein-Keshet L (1988) Mathematical Models in Biology. The Random House

Flach EH, Schnell S (2010) Stability of open pathways. Mathematical Biosciences 228(2):147 - 152

Fraser SJ, Roussel MR (1994) Phase-plane geometries in enzyme kinetics. Canadian Journal of Chemistry 72(3):800-812

Goelzer A, Brikci F, Martin-Verstraete I, Noirot P, Bessières P, Aymerich S, Fromion $\mathrm{V}$ (2008) Reconstruction and analysis of the genetic and metabolic regulatory networks of the central metabolism of bacillus subtilis. BMC systems biology 2(1):20

Heinrich R, Schuster S (1996) The regulation of cellular systems. Chapman \& Hall. New York. US

Jacquez JA, Simon CP (1993) Qualitative theory of compartmental systems. SIAM Review 35:43-79

Murray J (2002) Mathematical biology. Springer

Ndiaye I, Gouzé JL (2013) Global stability of reversible enzymatic metabolic chains. Acta Biotheoretica 61(1):41-57 
Oyarzún D, Chaves M, Hoff-Hoffmeyer-Zlotnik M (2012) Multistability and oscillations in genetic control of metabolism. Journal of Theoretical Biology 295:139_ 153

Perko L (2001) Differential equations and dynamical systems. Springer, Berlin

Segel L, Slemrod M (1989) The quasi-steady-state assumption: a case study in perturbation. SIAM review pp 446-477

Smith HL (1995) Monotone Dynamical Systems: An introduction to the theory of competitive and cooperative systems, vol 41. American Mathematical Soc. Mathematical surveys and monographs

Sontag E (2004) Some new directions in control theory inspired by systems biology. Syst Biol 1(1):10

Stephanopoulos GN, Aristidou A, , Nielsen J (1998) Metabolic engineering: principles and methodologies. Academic Press

Steuer R, Junker BH (2008) Computational models of metabolism: Stability and regulation in metabolic networks. In: Advances in Chemical Physics, John Wiley and Sons, pp 105-251

\section{A Monotone systems}

Monotone systems form an important class of dynamical systems, and are particularly well adapted to mathematical models in biology (Sontag, 2004), because they are defined by conditions related to the signs of Jacobian matrix. The reader may consult the references (Smith, 1995) for a review or an exhaustive presentation of the theory of monotone systems.

In summary, if the system is cooperative, then the flow preserves the partial order of trajectories in $\mathfrak{R}^{n}$ (the flow is monotone). Cooperativity is easy to check by looking at the signs of the elements of the Jacobian matrix, that should verify $\frac{\partial f_{i}}{\partial x_{j}}(t, x) \geq 0 \quad \forall i \neq j$. These systems have a strong tendency to converge to the set of their equilibria (Smith, 1995). Here we only need a simple proposition, easily deduced from Proposition 2.1 p. 34 of (Smith, 1995). The monotone system is defined on a convex set $X$.

Proposition 4 Let us suppose that only one equilibrium $x^{*}$ exists in $X$; if moreover it exists two points $x^{+}, x^{-}$in $X$ such that $f\left(x^{+}\right)<0$ and $f\left(x^{-}\right) \geq 0$, with $x^{-}<x^{*}<x^{+}$, then the hyperrectangle built by the two points $x^{-}, x^{+}$is invariant, and every solution in this rectangle converges toward the equilibrium point.

\section{B Matrices and Compartmental systems}

We introduce the notion of irreducibility of a matrix: we give one of the possible definition, which will be used in theorem 3.

\section{Definition 1 Irreducible Matrix}

A matrix is irreducible if its graph is strongly connected (there is a directed path from any compartment to any other compartment).

Let us now give a few reminders about compartmental systems (see (Jacquez and Simon, 1993)). This kind of models describes the dynamics of interconnected $n$-compartments.

Definition 2 Compartmental Matrix

Matrix $J$ is a compartmental $(n \times n)$ matrix if it satisfies the following three properties (Jacquez and Simon, 1993):

$$
J_{i i} \leq 0 \text { for all } i,
$$




$$
\begin{aligned}
& J_{i j} \geq 0 \quad \text { for } \text { all } i \neq j, \\
& -J_{j j} \geq \sum_{i \neq j} J_{i j} \text { for all } j
\end{aligned}
$$

There are also some theorems on the stability of linear and nonlinear compartmental systems (see (Jacquez and Simon, 1993)).

We recall some definitions and properties concerning output, see (Jacquez and Simon, 1993, p. 47) and (Bastin and Guffens, 2006).

Definition 3 Fully outflow connected network

A compartment $x_{i}$ is outflow (output) connected if there is a path $x_{i} \rightarrow x_{j} \rightarrow \ldots \rightarrow x_{l}$ from $x_{i}$ until a compartment $x_{l}$ with an outflow to the exterior of the system. The network is fully outflow connected if all compartments are outflow connected.

The following proposition is in (Jacquez and Simon, 1993, p. 52).

Proposition 5 Invertibility of a compartmental matrix

A compartmental matrix is regular if and only if the associated network is fully outflow connected.

Intuitively, it means that the system has no traps where the flows accumulate (see (Jacquez and Simon, 1993)). We recall that in this case the matrix has eigenvalues with negative real parts (Jacquez and Simon, 1993, p. 51), and the associated linear system is asymptotically stable. 